\title{
Preventing brain damage in necrotizing enterocolitis
}

\section{C6 \\ ...D-NAC- treated mice did not have impaired neurocognitive performance...

(N) impairments caused by necrotizing enterocolitis (NEC), in a mouse model that recapitulates the human disease, are caused by the effects of Toll-like receptor 4 (TLR4) activation on brain microglia and accumulation of reactive oxygen species (ROS), owing to the release of high mobility group protein $\mathrm{B} 1$ (HMGB1) from the intestinal epithelium. Oral treatment with a nanoparticle coupled to the antioxidant $\mathrm{N}$-acetyl-L-cysteine (NAC) helped to prevent the neurodegenerative effects, indicating a way to protect infants with NEC from cognitive impairment. \\ NEC affects $\sim 10 \%$ of premature infants and nearly half of those that survive the disease will develop neurodevelopmental defects. "The cognitive dysfunction in children with NEC is more severe than the impairment that occurs in premature infants who do not develop NEC, indicating that the intestinal dysfunction must be somehow related to the impaired brain function," explains senior author David Hackam. "This study was performed to unravel the causes of NEC-associated brain injury, and to provide an opportunity}

A new paper shows that cognitive

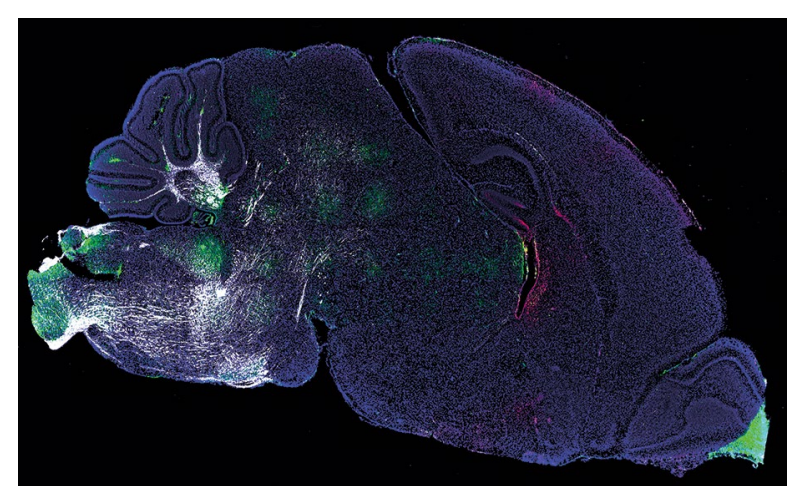

Brain of an 11-day-old mouse with NEC. The microglial activation marker IBA1 is stained green, myelin is stained white and ROS accumulation is shown in red by dihydroethidium staining. Image courtesy of D. F. Niño and D. J. Hackam, Johns Hopkins University, Baltimore, USA. to prevent the cognitive impairment that occurs in patients with NEC."

The team first validated their animal model of NEC, which comprises administration of formula feeds, hypoxia and stool from a child with severe NEC to newborn mice. The protocol resulted in disruption of the intestinal villus architecture and increased expression of $I L 1 B$. In addition, mouse brains showed impaired myelination and decreased overall total volume. Cognitive function tests in mature mice that had been exposed to experimental NEC revealed marked impairment of neurocognitive performance. These findings resemble the features of NEC in humans.

Exploring possible underlying mechanisms, the team found decreased expression of ionized calcium-binding adapter molecule 1 (IBA1) in brains of infants and mice with NEC, indicating microglial activation. In addition, brain sites of ROS accumulation coincided with those of reduced myelin basic protein (MBP) expression, and microglial activation was associated with loss of oligodendrocyte progenitor cells.

Previous work has shown that microglia activation can be triggered via TLR4; hence, the team created mice lacking microglial TLR4 expression. Following exposure to the NEC induction protocol, these mice did not develop NEC-associated brain injury, shown by levels of IBA1 expression, ROS accumulation, MBP expression and myelination patterns, which were similar to those of wild-type mice.

Release of the TLR4 ligand HMGB1 by the intestinal epithelium has previously been shown in mice and humans with NEC. Applying their NEC induction protocol to mice lacking HMGB1 in the intestinal epithelium, the team found reduced microglial activation (IBA1 expression) and ROS accumulation, as well as preserved MBP expression, compared with wild-type mice. The possible central role of HMGB1 was further corroborated in wildtype mice with NEC that received intranasal anti-HMGB1 antibodies and in wild-type mice that received injections of recombinant HMGB1.

As the NEC-associated brain injury seemed to depend on TLR4 activation and ROS accumulation, the researchers tested the effects of administering the antioxidant NAC coupled to dendrimers (D-NAC). These nanoparticles have been shown to localize to activated microglia. In their NEC mouse model, oral administration of D-NAC resulted in return of brain injury marker levels to those of wild-type mice. In addition, D-NAC-treated mice did not have impaired neurocognitive performance according to functional tests. Notably, beneficial effects of D-NAC treatment were still observed 60 days after initial intestinal injury.

"These studies reveal that TLR4 activation leads to HMGB1 release, which travels to the brain and activates TLR4 on the microglia resulting in brain injury," concludes Hackam. "The delivery of dendrimers tagged with a ROS scavenger can prevent NEC-associated brain injury; we plan to extend this work to patients in clinical trials."

Clemens Thoma

ORIGINAL ARTICLE Niño, D. F. et al. Cognitive impairments induced by necrotizing enterocolitis can be prevented by inhibiting microglial activation in mouse brain. Sci. Transl. Med. 10, eaan0237 (2018) FURTHER READING Niño, D. F. et al. Necrotizing enterocolitis: new insights into pathogenesis and mechanisms. Nat. Rev. Gastroenterol. Hepatol. 13, 590-600 (2016) 